PDFlib PLOP: PDF Linearization, Optimization, Protection

Page inserted by evaluation version www.pdflib.com - sales@pdflib.com 


\title{
Genetic Aspects of Abdominal Aortic Aneurysm
}

\author{
ALAIN VERLOES,,$^{a, b}$ NATZI SAKALIHASAN, ${ }^{c}$ \\ RAYMOND LIMET, ${ }^{c}$ AND LUCIEN KOULISCHER ${ }^{a}$ \\ ${ }^{a}$ Centre for Human Genetics \\ 'Department of Cardiovascular Surgery \\ Liège University \\ CHU Sart Tilman \\ B-4000 Liège, Belgium
}

\section{INTRODUCTION}

Abdominal aortic aneurysm (AAA) is a common pathology, with an estimated incidence of 20 to 40 cases $/ 100,000$ persons per year, ${ }^{1,2}$ and its prevalence in adult autopsy series lies between 1 and $6 \% .^{3}$ In a recent English study, its prevalence is $2.6 \%$ in men aged 60 to $64,6 \%$ for those aged 65 to 74 and $9 \%$ over $75 .{ }^{4}$ By ultrasound screening, Collin et al..$^{5}$ found an abdominal aortic aneurysm in $5.4 \%$ of men aged 65 to $74,2.3 \%$ of them being $4 \mathrm{~cm}$ or more in diameter. In an epidemiological survey held in 1992 in England and Wales, AAA caused $1.9 \%$ of all deaths in men and $0.7 \%$ of all deaths in women aged 60 or over. ${ }^{6}$ The etiologies of aneurysmal dilatation are multiple. Some well-known causes are uncommon (Marfan syndrome, Ehlers-Danlos disease type IV, fibromuscular dysplasia) or historical (syphilis). The frequent coincidence of atherosclerosis and the presence of an abdominal aortic aneurysm has led to the opinion that the aneurysm might be a consequence of atherosclerosis. Some facts derived from the clinical observation are, however, incompatible with a direct causal relationship between aneurysms and atherosclerosis? and suggest that atherosclerosis is either coincidental or facilitating. AAA is frequent in the elderly. The familial clustering of cases has only recently drawn attention. Since the first case report by Martin Clifton, in $1977,{ }^{8}$ several series have confirmed AAA as one of the most frequent "familial" diseases. It appears probable that "not everyone is prone to aneurysm" and that a particular genetic character predisposes the aging arterial wall to an aneurysmal transformation, perhaps in response to an accidental factor. Although some descriptive statistics are available on the familial aspects and on the natural history of the disease, the pathogeny and the genetic background still remain largely obscure, and each mode of inheritance (dominant, recessive, X-linked, multifactorial) has been advocated. ${ }^{9}$ We shall review here the data on familial clustering of AAA.

\footnotetext{
${ }^{b}$ Corresponding author.
} 


\section{AAA: WHO IS AFFECTED?}

In familial studies, persons have to be assigned a status: affected, unaffected, or unknown. Whereas this is of little concern for congenital, qualitative defects, the determination of the affection status is a major difficulty in the study of AAA families.

An aneurysm can be defined as an abnormal, permanent, and irreversible localized dilatation of a vessel. If this definition allows straightforward diagnosis of large aneurysms, it is ambiguous for the smaller ones. The precision of the definition is important to decide what is considered or rejected as infrarenal AAA, and may have unsuspected effects on segregation analysis and epidemiological studies. ${ }^{10}$ Some clinicians consider that an aneurysm is present when the largest axis of the infrarenal aorta exceeds an absolute diameter ranging from 3 to $4 \mathrm{~cm}$, or when dilatation exceeds $50 \%$ of the diameter of the normal aorta for the sex and the height of the patient. Others use a ratio of the diameters of the infrarenal and suprarenal aorta exceeding a threshold varying from $1.5: 1$ to $2: 1$.

Aneurysms are pathological dilatations of vessels progressively worsening with time, thus delayed in their appearance. They can affect any arterial segment of the organism but they are most often seen at the arterial structural weak points: transition zones between the fixed aorta and the free aorta and flexion zones. They can be unique or multiple in the same patient. The majority of clinically detectable aneurysms are found in the infrarenal abdominal aorta. They often extend along the common iliac artery, and, more rarely, on the external iliac artery. Aneurysms of the popliteal arteries are frequently associated with aneurysms of the infrarenal aorta, and aneurysms of cerebral arteries are also observed with an increased frequency in patients or relatives of AAA patients ${ }^{11}$ (6 of 313 in our experience). In AAA patients, there could be a generalized tendency to peripheral artery dilatation. ${ }^{12}$ The delayed appearance, the multiplicity of sites, and the metachronous development of arterial aneurysms obviously complicate the status assignment of individuals in pedigree studies and the comparison between series.

\section{SEEKING POPULATION-BASED EPIDEMIOLOGICAL DATA}

Despite the huge number of publications on AAA, we still lack detailed epidemiological data on nonruptured AAA. Bickerstaff $e t$ al. ${ }^{1}$ computed incidences of AAA by age group in a general population. Their values have been converted to cumulative incidences by Majumder et al. ${ }^{13}$ Data were collected between 1951 and 1980 , and concern the predominantly white population of Rochester, Minnesota. AAA patients were gathered from all sources, including diagnosis "by chance" and necropsic discovery of unsuspected patients, but not on ultrasound (US) scan, so that underestimation is still likely. All cases of ruptured AAA were collected by Bengtsson for the period 1971-1986 in Malmö, Sweden. ${ }^{14}$ Other recent epidemiological studies are available, possibly showing a trend toward an increase in age-standardized incidence of AAA in northern Europe. ${ }^{2,15,16}$ Whether this reflects a true modification in the incidence, or illustrates better awareness 
and better (and earlier) diagnosis thanks to modern imaging techniques is not certain, but represents a real problem for all population-based genetic analysis.

\section{AAA IN SIBSHIPS: THE EMERGENCE OF A FAMILIAL DISORDER}

Clifton, ${ }^{8}$ reporting three affected brothers in 1977 , pioneered the study of familial aggregation of AAA. Johansen and Koepsell ${ }^{17}$ compared the family histories of 250 patients with AAA to those of 250 control subjects. Among the latter, $2.4 \%$ reported a first-degree relative with an aneurysm, compared with $19.2 \%$ of the patients with AAA, representing a 11.6-fold increase in risk among persons with an affected first-degree relative. Darling et al. ${ }^{18}$ found $15.1 \%$ of proband with an affected first-degree relative as compared to $1.8 \%$ of a control group of 500 patients of similar age and sex. Several other series were published, ${ }^{17,19-22}$ including our own systematic analysis of 313 pedigrees. ${ }^{23}$ The series are compared in TABLE 1. The sex ratio in favor of males varies between $4: 1$ and $20: 1$. Based on nonsystematic clinical diagnosis, the proportion of AAA proband with a positive family history goes from 12 to $20 \%$, and the relative risk of AAA for siblings was 10 to $20 \%$. Webster et al. ${ }^{7}$ found $16.2 \%$ of familial AAA history by anamnesis of 43 consecutive patients. After prospective US screening, the number of familial cases rose to $27.9 \%$. Bengtsson et al. ${ }^{24}$ found $29 \%$ of AAA in male and $6 \%$ in female relatives after US screening, confirming the extremely high incidence of cryptic, asymptomatic familial AAA. These discrepancies leave much room for doubt as to the accuracy of older epidemiological studies of AAA. Differences in natural history of AAA depending on the familial history were addressed in larger series. In the Darling et al. study, ${ }^{18}$ no significant difference was found between the patients with nonfamilial and familial AAA in anatomic extent, or associated occlusive disease. Patients with familial AAA were more likely to be women (35 vs. $14 \%$ ), and, men, in their group, were affected about five years earlier than women.

Table 1. Comparison of Familial Studies of

\begin{tabular}{lccc}
\hline & Norrgard & Tilson & Johansen \\
\hline Pedigrees & & & \\
$N$ & $\mathbf{8 7}$ (initially 200) & 50 & 250 \\
Multiplex & $18(20.6 \%)$ & 50 & $48(19.2 \%)$ \\
Horizontal & 10 & 28 & 18 \\
Vertical/complex & 8 & 22 & $\geq 19$ \\
AAA & 103 & 127 & $\geq 307$ \\
AAA (familial subgroup) & 38 & 127 & $\geq 105$ \\
Sex ratio (M/F) & $155: 45(3.75: 1)$ & $?$ & $207: 43(4.81: 1)$ \\
Familial & $30: 8(3.75: 1)$ & $11: 16(6.94: 1)$ & $?$ \\
Sporadic & $?$ & $?$ & $?$ \\
Age at diagnosis & $67($ M66/F70) & $?$ & $?$ \\
Familial & $65(n=19)$ & $?$ & $?$ \\
Sporadic & $?$ & $?$ & $?$ \\
Rupture rate & $68 / 200($ initial) & $?$ & $?$ \\
Familial & $14 / 38(36.8 \%)$ & $?$ & $?$ \\
Sporadic & $?$ & $?$ & $?$ \\
\hline
\end{tabular}


The risk of rupture was strongly correlated with familial disease and the presence of a female member with aneurysm ( 63 vs. $37 \%$ ). In our own study of 313 pedigrees, we found similar differences: the age at diagnosis two years earlier $(p<0.05)$ and the age at rupture 10 years earlier $(p<0.001)$ in males of multiplex pedigrees, as well as the rupture rate were higher in the latter ( 32.4 vs. $8.7 \%)$. Ethnic differences in AAA have been considered by Johnson et al..$^{25}$ in a survey of autopsies and abdominal CT scans: white males have a frequency of abdominal aortic aneurysm about three times that in black males, black females, or white females; all three of the latter groups had comparable frequencies.

\section{AAA IS A GENETIC DISEASE}

Genetic aspects of AAA have been the subject of very few studies. Because of the irregular clustering of cases, some authors inferred it was multifactorial. ${ }^{26}$ Tilson and Seashore ${ }^{20}$ presented 50 families, including three pairs of identical twins. Those families were collected by various teams, and no data were given either on the mode of ascertainment or on the isolated AAA from the same population. Twenty-nine of 50 were single-generational, 18 showed simple "vertical" transmission, and 3 were "complex." In an empirical approach to the results, they favored a frequent X-linked dominant form and a less common autosomal dominant, or a multifactorial model. Norrgard et al. ${ }^{19}$ illustrated several pedigrees.

\section{Familial AAA Does Not Respect Simple Pattern}

Whereas the determination of a mode of inheritance does not require sophisticated analysis for rare, fully penetrant, qualitative disorders of early onset (such as cystic fibrosis or hemophilia), it can be a real problem for a common disorder

Abdominal Aortic Aneurysm

\begin{tabular}{|c|c|c|c|}
\hline Cole & Darling & Webster & Verloes \\
\hline 305 & 542 & 91 & 313 \\
\hline $37(12.1 \%)$ & $82(15.1 \%)$ & $14(15.3 \%)$ & $39(12.4 \%)$ \\
\hline 18 & $?$ & 11 & 23 \\
\hline 19 & ? & 3 & 16 \\
\hline$?$ & 669 & 108 & 357 \\
\hline 91 & 209 & 31 & 81 \\
\hline$?$ & $532: 137(3.88: 1)$ & $49 / 19(4.68: 1)$ & $340 / 17(20: 1)$ \\
\hline $56: 35(1.6: 1)$ & $136: 73 \quad(1.86: 1)$ & $20: 10(2: 1)$ & $76 / 5 \quad(15: 1)$ \\
\hline ? & $396: 64 \quad(6.19: 1)$ & $69: 9(7.67: 1)$ & $264: 12(22: 1)$ \\
\hline ? & ? & M67.1/F69.2 & M66.2/F69.5 \\
\hline$?$ & M62.4/F71.2 & $?$ & M65/F73 \\
\hline ? & M67.8/F68.8 & ? & M66.6/F68 \\
\hline ? & $?$ & $?$ & $52 / 357(14.6 \%)$ \\
\hline $22 / 52(42 \%)$ & $42 / 209(20.1 \%)$ & ? & $29 / 81 \quad(35.8 \%)$ \\
\hline ? & $?$ & $?$ & $20 / 276 \quad(8.3 \%)$ \\
\hline
\end{tabular}


with irregular familial expression. Irregular familial aggregation may result from partially and/or age-dependent penetrance of one or several rare dominant, or frequent recessive genes (Mendelian inheritance patterns), from additive effects of several genes (polygenic inheritance) or environmental factors, or from a combination of those effects (multifactorial inheritance). Then, the question whether a single identifiable locus accounts for a significant amount of the phenotypic variation in a population may be addressed through the methodology of segregation analysis.

\section{Experimental Approach}

The Blotchy mouse ${ }^{27}$ shows a decreased resistance of the skin and a propensity to AAA related to a mutation on chromosome $X$. This deficiency in cross-linking of collagen and elastin is biochemically related to a decrease in the conversion of lysine to lysine-aldehyde residues. This congenital biochemical defect is close to the pathology of aneurysms produced in the turkey ${ }^{28}$ and mouse $\mathrm{e}^{29}$ by administration of lathyrogens, which prevent the formation of aldehydes by inactivating lysyl oxidase. Experimental deficiency in copper, which is a cofactor of lysyl-oxidase, can induce aneurysms in the pig and in the chicken. Although those experiments are significant models for inborn defects in AAA formation, caution is advisable before extrapolation to the human condition for two reasons. First, the aneurysmal transformation takes several decades in man whereas in animal models it requires a relatively short time; second, the composition of the aorta in terms of fibrous protein is not perfectly similar in man and animals.

\section{Formal Genetics Approach}

Most studies of common diseases assume that genetically determined factors are numerous and give an equal and individually small contribution to the phenotype. In polygenic models, a quantitative trait (with threshold) or a qualitative trait result from additive effects of several biallelic $(A, a)$ loci. Each locus has an even-weighted effect. Loci are unlinked (independent transmission), and heterozygotes $(A a)$ contribute an effect which is intermediate between the effects of both types of homozygotes $(A A, a a)$. Each locus accounts for a part in the genetic variance of the population. The parent-child and child-child correlations are $50 \%$ for each locus. In those models, formal genetic analysis is limited to the computation of heritability, which is the proportion of total phenotypic variance due to polygenic effects. Assuming a multifactorial model, Powell and Greenhalgh ${ }^{26}$ calculated a heritability of $70 \%$, using Falconer's method, for a series of 60 patients, 25 of them with positive familial history.

Segregation analysis is basically the comparison of the observed proportion of affected offspring with the expected proportion according to a particular genetic hypothesis. To assess evidence of a major gene effect in the presence of other familial sources of correlation (polygene, sociocultural factors, etc.), pedigrees may be analyzed using the pointer strategy. ${ }^{30}$ This methodology was developed 
as a tool for analysis of multigenerational pedigrees and is based on the mixed model, a mathematical model combining genetic and multifactorial components. ${ }^{31-33}$ It includes modifiable parameters, which allow the definition of several submodels (purely dominant, multifactorial, etc.). The likelihood of the observed sibships conditional on the phenotype of the parents is computed and maximized through fine-tuning of those transmission parameters. The likelihood of the general model (mixed) is compared with appropriate statistical tests to restricted submodels, the best model being the more likely, or, for nonsignificantly differing submodels, the most parsimonious in terms of parameters. Cumulative incidence of the trait in the population (stratified, if necessary, for age and sex in several subgroups) and ascertainment probability of the sample must be known. It implies that reliable epidemiological data are available for the studied population. Genetic heterogeneity cannot be directly addressed by this mode of analysis.

Two teams have attempted segregation analysis of AAA. In Majumder et al.'s work, ${ }^{7.13 .22}$ data on first-degree relatives of 91 probands from 91 pedigrees were collected (sex ratio 79:12). Cases with patent AAA (thus probably $>50 \mathrm{~mm}$ ) were considered as positive. Their number of multiplex pedigrees was relatively small (13 of 91). The most likely genetic model was an autosomal diallelic locus with a recessively inherited gene for AAA. A multifactorial component did not increase significantly the likelihood of the data set. In our own analysis of 324 probands (sex ratio 312:12) from 313 pedigrees (including 39 multiplex families), we used a less drastic definition: infrarenal/suprarenal ratio $>1.5$ or absolute diameter $>30 \mathrm{~mm}$. Our analysis favored a simple autosomal dominant model, with a carrier frequency of 1 of 250 and a age-related penetrance never higher than 0.4 , even in the subgroup of elder men. Like Majumder et al., we noted that a multifactorial component did not lead to a significantly better fit. Whether differences in the inheritance mode between the two studies come from differing ascertainment, variable definitions of the affected status, or differing local epidemiology (suspected on the basis of our unusually high sex ratio) remains unknown. Nevertheless, the importance of a single genetic factor in the appearance of AAA remains the most important lesson resulting from those formal genetic works. The difference between sexes may nevertheless indicate that an adjuvant factor (hypertension, atheromatosis) may act as a modifier on penetrance and/or expressivity of the disease.

\section{Candidate Gene Approach}

Both clinical considerations and experimental results allow suspect genes involved in the constitution of the normal matrix and in its turnover to play a role in the genetic background of aneurysmal predisposition. Among structural components of the conjunctive intercellular matrix are collagens, genes of the matrix metalloproteinases family (including the chromosome 11q22-23 collagenase cluster members), the tissue inhibitors of metalloproteinases), and two genes, HP and CETP, that map in the vicinity of CLG4A (although the latter two are not primarily involved in matrix metabolism ${ }^{34}$ ). They are listed in TABLE 2. 
TABLE 2. Candidate Mapped Genes for Abdominal Aortic Aneurysm

\begin{tabular}{|c|c|c|}
\hline Gene & Synonyms Gene Symbol(s) & $\begin{array}{l}\text { Chromosome } \\
\text { Location }\end{array}$ \\
\hline \multicolumn{3}{|l|}{ Matrix proteins } \\
\hline Al-chains of collagen type I & COLIA1 & $17 q 22$ \\
\hline A2-chains of collagen type I & COL1A2 & $7 q 22.1$ \\
\hline A-chain of collagen type III & COL $3 \mathrm{Al}$ & $2 q 31$ \\
\hline Fibrillin 1 & FBN1 & $15 q 21.1$ \\
\hline Elastin & ELN & $7 q 11.2$ \\
\hline Collagenase A type IV & $\begin{array}{l}\text { MMP2 } 72 \mathrm{kDa} \text { gelatinase } \\
\text { CLG4A }\end{array}$ & $16 q 13$ \\
\hline \multicolumn{3}{|l|}{ Degradation enzymes } \\
\hline Gelatinase B & $\begin{array}{l}\text { MMP9 } 92=k D a \text { gelatinase } \\
\text { CLG4B }\end{array}$ & $20 q 11-13$ \\
\hline Interstitial collagenase & CLG/MMPI & $11 q 22-23$ \\
\hline Neutrophil collagenase & CLG1/MMP8 & $11 q 21-22$ \\
\hline Matrilysin & PUMP1/MMP7 & $11 q 22-24$ \\
\hline Collagenase 3 & CLG3/MMP13 & $11 \mathrm{q} 22-23$ \\
\hline Stromelysin 1 & STMY/MMP3 & $11 q 22-23$ \\
\hline Stromelysin 2 & STMY2/MMP10 & $11 q 22-23$ \\
\hline \multicolumn{3}{|l|}{ Degradation inhibitors } \\
\hline Metalloproteinase inhibitor type 1 & TIMP1/HCI/EPA & Xp11.1 \\
\hline Metalloproteinase inhibitor type 2 & TIMP2 & $17 q 25$ \\
\hline \multicolumn{3}{|l|}{ Chromosome 16 candidates } \\
\hline Cholesterol-ester transfer protein & CETP & $16 \mathrm{q} 21$ \\
\hline Haptoglobin alpha chain & HP & $16 q 21$ \\
\hline
\end{tabular}

\section{Collagen Genes}

All cell types of the vascular wall are actively engaged in the synthesis of collagen. Collagens types I and III are the main varieties found in the arterial wall. Collagen IV is found in basal membranes. Defects of collagen I chains are well known in all forms of osteogenesis imperfecta, in which no vascular problem arises. Type III collagen is localized in the subendothelial space, and both types I and III are present in the media. In human thoracic aorta, abdominal aorta, and carotid artery approximately $50-75 \%$ of total collagen in the media consists of type I collagen. Interstitial collagens type I and III (and the minor type V) are synthesized by the fibroblasts of the adventitia. The post-translational hydroxylation of some prolyl residues allows the association of three $\alpha$-chains in a stable triple helix structure that subsequently polymerize spontaneously to form the "interstitial fibers," highly resistant to proteolysis. Type I and type III collagens make hybrid fibers surrounding a central core of type V. Other types of collagen have also been described in the vascular wall: type VI collagen and type VIII (which could serve as a bridge between the collagen fibers and the noncollagen components). Each of those constituents of the collagen bundles may be a candidate for some cases of familial AAA.

Loosemore et al. ${ }^{35}$ suggested that a deficiency of type III collagen might be the basis for the aneurysm formation. Menashi et al. ${ }^{36}$ showed a low content of type III collagen in a group of patients with familial AAA. Minor normal allelic 
variants of COL $3 \mathrm{Al}$ were found in association with AAA. A less elastic aneurysm wall was found in those patients. ${ }^{37}$ Thermally unstable procollagen III was found in two patients with multiple aneurysms. ${ }^{38}$ Furthermore, mutations of collagen type III are known to occur in the vascular type IV of Ehlers-Danlos (EDIV). ${ }^{39}$ Clinical hallmarks of EDIV include facial dysmorphism (pinched nose, thin lips), fragile, easily bruisable, translucent skin with prominent venous markings, mitral valve prolapse, microangiopathy of skin capillaries with microbleedings and microaneurysms, tortuous arteries, AAA, and very commonly spontaneous arterial and bowel ruptures. In many families, EDIV is due to mutations of pro-alphachains of type III collagen.

In 1990, Sirpa Kontusaari showed two single base mutations in COL 3A1 in two families with dominantly inherited AAA. In the first family, the mutation was shown to result in an amino acid substitution (GLY619ARG)..$^{40}$ In the second, presenting with AAA and easy bruisability, a single base mutation in intron 20 $\left(G^{+1 \text { IVS20 }}\right)^{41}$ was shown to induce aberrant splicing of the mRNA, hence reducing the synthesis of the $\alpha$ (III) chain. This family is of peculiar clinical importance, as clinical presentations extended from the classical EDIV disease to isolated AAA. Tromp et al..$^{42}$ carried out detailed DNA sequencing of the triple-helical domain of type III procollagen on CDNA from 54 patients with aortic aneurysms, 43 of them with at least one affected relative. Only two amino acid substitutions were found (GLY136ARG, THR501PRO), the second of unclear functional significance, thus indicating that collagen III mutations are not a major cause of common AAA.

\section{Elastin and Fibrillin}

Elastin is a fibrous protein, displaying a distribution in the vascular walls more restricted than that of collagen. The elastin fibers, by their abundance and their elasticity, are one of the most important constituents of the arterial wall. The smooth muscle cells synthesize medial elastin. It is organized in concentric lamellae in the media of the large arteries found near the heart, such as the aorta or pulmonary artery (so-called elastic arteries). The number of concentric lamellae appears to be genetically determined and a progressive decrease in their number is observed from the heart to the aorto-iliac bifurcation. Elastin is dramatically reduced in the AAA wall. ${ }^{43}$ Tropoelastin, the soluble precursor of elastin, is rich in lysine, which is oxidized by a lysyl-oxidase into reactional aldehydes that condense to form desmosine and isodesmosine cross-links between four molecules. Without this bridging, elastin could not be elastic. The tropoelastin molecules are deposited into a microfibrillar structure containing fibrillin as the main component.

Some types of ducks, particularly sensitive to aneurysmal transformation, are characterized by a lysyl-oxidase defect. Reduced synthesis of elastin may result from large mutations or larger deletions of the elastin gene region on chromosome 7. In patients with deletions limited to the ELN gene, the phenotype is autosomal dominant supravalvular aortic stenosis, ${ }^{44.45}$ whereas larger deletions led to the Williams-Beuren ("elfin facies") syndrome. ${ }^{46}$ None of those phenotypes is associated with AAA, but nothing is known about the phenotypic effect of missense 
mutations of the elastin gene (which could interfere with cross-linking of tropelastin). Mutations in the fibrillin-1 gene are present in almost all families with Marfan syndromes, ${ }^{47,48}$ a well-known disorder characterized, on the vascular field, by dilatation of the aortic root (prone to dissection or rupture) and valvular insufficiency, but not by classical AAA. Nevertheless, the full spectrum of phenotypes associated with mutations of fibrillin-1 is still to be defined.

\section{Collagenases}

Collagenases are members of a group of secreted zinc metalloproteases, which degrade the collagens and other constituents of the extracellular matrix. Thus, increased activity, abnormal regulation or ectopic expression of those genes could affect the turnover of collagen fibrils. CLG4A, located on chromosome 16, is secreted from normal skin fibroblasts, whereas CLG4B, recently mapped on chromosome $20,{ }^{49}$ is produced by normal alveolar macrophages and granulocytes. Both have closely resembling structures, with 13 exons including a fibronectinlike domain, ${ }^{50}$ and are active on the basement membrane collagen IV. CLG4B digests collagen types I, II, III, IV, and V, denatured collagen (gelatin), and elastin. At least four collagenase genes are located on chromosome 11: CLG1, CLG3, STMY, and STMY2. ${ }^{51-53}$ CLG1 is the only enzyme to initiate breakdown of collagens I, II, and III. Association has been observed between the CLG4A region and AAA, but until now, no published linkage studies sustain a role of the other collagenases in the etiology of familial AAA. In a recent study, St Jean did not find disequilibrium linkage between an intragenic CLG4B microsatellite marker and the presence of AAA.99

\section{CONCLUSION}

AAA is a complex disorder with multiple risk factors and probably multiple pathogenetic pathways. The major determinant factors in the appearance of AAA appear to be genetic and behave usually as dominant traits. Several inborn defects of connective tissue matrix are candidates for AAA, although, until now, only a limited fraction of families have been shown to be linked to mutations of the COL3A1 gene. Linkage studies in selected pedigrees should lead to the unraveling of the basic pathogenesis in the near future.

\section{REFERENCES}

1. Bickerstaff, L. K., L. H. Hollier, H. J. Van Peenen, L. J. Melton, P. C. PairoLERo \& K. J. CherRy. 1984. Abdominal aortic aneurysm: The changing natural history. J. Vasc. Surg. 1: 6-12.

2. Melton, L. J., L. K. Bickerstaff, L. H. Hollier, H. J. Van Peenen, J. T. Lie, P. C. Pairolero \& K. J. Cherry. 1984. Changing incidence of abdominal aortic aneurysms: A population-based study. Am. J. Epidemiol. 120: 379-386.

3. FIEVEZ, M. 1989. Aneurysms and arterial dissections. In Diseases of the arterial wall. 
J. P. Camilleri, L. B. Colin, J. P. Fiessinger \& J. Bariety, Eds.: 533-545. Springer Verlag. New York.

4. Twomey, A., E. Twomey, R. A. Wilkins \& J. D. Lewis. 1986. Unrecognised aneurysmal disease in male hypertensive patients. Int. Angiol. 5: 269-273.

5. Collin, J., L. Araujo, J. Walton \& D. Lindsell. 1988. Oxford screening programme for abdominal aortic aneurysm in men aged 65 to 74 years. Lancet 2: 613-615.

6. Poulter, N. 1993. Abdominal aortic aneurysm. Lancet 341: 215-220.

7. Webster, M. W., R. E. Ferrell, P. L. St Jean, P. P. Majumder, R. S. Fogel \& D. L. STEED. 1991. Ultrasound screening of first-degree relatives of patients with abdominal aortic aneurysm. J. Vasc. Surg. 13: 9-13.

8. Clifton, M. A. 1977. Familial abdominal aortic aneurysms. Br. J. Surg. 64: 765-766.

9. Tilson, M. D. \& M. R. Seashore. 1984. Human genetics of abdominal aortic aneurysm. Surg. Gynecol. Obstet. 158: 129-132.

10. Moher, D., C. W. Cole \& G. B. HiLl. 1992. Epidemiology of abdominal aortic aneurysm: The effect of differing definitions. Eur. J. Vasc. Surg. 6: 647-650.

11. Norrgard, Ö., K. A. Ängqvist, H. Fodstad, A. Forssell \& M. Lindberg. 1987. Coexistence of abdominal aortic aneurysms and intracranial aneurysms. Acta Neurochir. 48: 164-170.

12. WARD, A. S. 1992. Aortic aneurysmal disease: A generalized dilating diathesis? Arch. Surg. 127: 990-991.

13. Majumder, P. P., P. L. St Jean, R. E. Ferrell, M. W. Webster \& D. L. Steed. 1991. On the inheritance of abdominal aortic aneurysm. Am. J. Hum. Genet. 48: 164-170.

14. Bengtsson, H. \& D. Bergqvist. 1993. Ruptured abdominal aortic aneurysm: A population-based study. J. Vasc. Surg. 18: 74-80.

15. Fowkes, F. G. R., C. C. A. MackINTYRe \& C. V. Ruckley. 1989. Increasing incidence of aortic aneurysm in England and Wales. BMJ 298: 33-35.

16. Bengtsson, H., D. Bergqvist \& N. H. SternBy. 1992. Increasing prevalence of abdominal aortic aneurysms, a necropsy study. Eur. J. Surg. 158: 19-23.

17. Johansen, K. \& T. Koepsell. 1986. Familial tendency for abdominal aortic aneurysms. JAMA 256: 1934-1936.

18. Darling, R. C., III. D. C. Brewster, R. C. Darling, G. M. LaMuraglia, A. C. Moncure, R. P. Cambria \& W. M. Abbott. 1989. Are familial abdominal aortic aneurysms different? J. Vasc. Surg. 10: 39-43.

19. Norrgard, O., O. Rais \& K. A. Angquist. 1984. Familial occurrence of abdominal aortic aneurysms. Surgery 95: 650-656.

20. Tilson, M. D. \& M. R. SEAshore. 1984. Fifty families with abdominal aortic aneurysms in two or more first-order relatives. Am. J. Surg. 147: 551-553.

21. Cole, C. W., G. G. Barber, A. G. Bouchard, C. Roberge, W. G. Waddell \& J. L. WELLINGTON. 1989. Abdominal aortic aneurysm: Consequences of a positive family history. Can. J. Surg. 32: 117-120.

22. Webster, M. W., P. L. St Jean, D. L. Steed, R. E. Ferrell \& P. P. Majumder. 1991. Abdominal aortic aneurysm: Result of a family study. J. Vasc. Surg. 13: 366-372.

23. Verloes, A., N. Sakalihasan, L. Koulischer \& R. Limet. 1995. Aneurysms of the abdominal aorta: Familial and genetic aspects in three hundred thirteen pedigrees. J. Vasc. Surg. 21: 646-655.

24. Bengtsson, H., O. Norrgard, K. A. Angquist, O. Ekberg, L. Oberg \& D. BergQVisT. 1989. Ultrasonographic screening of the abdominal aorta among siblings of patients with abdominal aortic aneurysms. Br. J. Surg. 76: 589-591.

25. Johnson, G., JR., A. Avery, E. G. McDougal, S. J. Burnham \& B. A. Keagy, 1985. Aneurysms of the abdominal aorta: Incidence in blacks and whites in North Carolina. Arch. Surg. 120: 1138-1140.

26. Powell, J. T. \& R. M. Greenhalgh. 1987. Multifactorial inheritance of abdominal aortic aneurysm. Eur. J. Vasc. Surg. 1: 29-31.

27. ANDREws, E. J., W. J. White \& L. P. Bullock. 1975. Spontaneous aortic aneurysms on blotchy mice. Am. J. Pathol. 78: 199. 
28. Gresham, G. A. \& A. N. Howard. 1961. Aortic rupture in the turkey. J. Atheroscler. Res. 1: 75-80.

29. Brophy, C. M., J. E. Tilson \& I. M. Braverman. 1988. Age of onset pattern of distribution and histology of aneurysm development in a genetically predisposed mouse model. J. Vasc. Surg. 8: 45-48.

30. Morton, N. E. \& J. M. Lalouel. 1983. Segregation analysis of familial data. In Methods in Genetic Epidemiology. N. E. Morton, D. C. Rao \& J. M. Lalouel, Eds: 62-102. Karger. Basel.

31. Elston, R. C. \& J. A. Stewart. 1974. A general model for the genetic analysis of pedigree data. Hum. Hered. 21: 523-542.

32. Morton, N. E. \& C. J. MCLean. 1974. Analysis of family resemblance. III. Complex segregation of quantitative traits. Am. J. Hum. Genet. 26: 489-503.

33. Lalouel, J. M. \& N. E. Morton. 1981. Complex segregation analysis with pointers. Hum. Hered. 31: 312-321.

34. Powell, J. T., A. Bashir \& S. Dawson. 1990. Genetic variation on chromosome 16 is associated with abdominal aortic aneurysm. Clin. Sci. 78: 13-16.

35. Loosemore, T. M., A. H. Child \& J. A. Dormandy. 1988. Familial abdominal aortic aneurysms. J. R. Soc. Med. 81: 472-473.

36. Menashi, S., J. S. Campa, R. M. Greenhalgh \& J. T. Powell. 1987. Collagen in abdominal aortic aneurysm: Typing, content and degradation. J. Vasc. Surg. 6: 578-582.

37. Powell, J. T., J. Adamson, S. T. R. McSweeney, R. M. Greenhalgh, S. E. Humphries \& A. Henney. 1991. Genetic variants of collagen III and abdominal aortic aneurysm. Eur. J. Vasc. Surg. 5: 145-148.

38. Deak, S. B., J. J. Ricotta, T. J. Mariani, S. T. Deak, M. A. Zatina, J. W. Mackenzie \& C. D. BoyD. 1992. Abnormalities in the biosynthesis of type III procollagen in cultured fibroblasts from two patients with multiple aneurysms. Matrix 12: 92-100.

39. Superti-Furga, A., B. Steinmann, F. Ramirez \& P. H. Byers. 1989. Molecular defects of type III procollagen in Ehlers-Danlos syndrome type IV. Hum. Genet. 82: $104-108$.

40. Kontusaari, S., G. Tromp, H. Kulvaniemi, A. M. Romanic \& D. J. Prockop. 1990. A mutation in the gene for type III procollagen (col 3Al) in a family with aortic aneurysms. J. Clin. Invest. 86: 1465-1473.

41. Kontusaari, S., G. Tromp, H. Kulvaniemi, R. L. Ladda \& D. J. Prockop. 1990. Inheritance of a RNA splicing mutation $(G+1 / V S 20)$ in the type III procollagen gene (COL3A1) in a family having aortic aneurysms and easy bruisability: Phenotypic overlap between familial arterial aneurysms and Ehlers-Danlos syndrome type IV. Am. J. Hum. Genet. 47: 112-120.

42. Tromp, G., Y. Wu, D. J. Prockop, S. L. Madhatheri, C. Kleinert, J. J. Earley, J. Zhuang, O. Norrgard, R. C. Darling, W. M. Abbott, C. W. Cole, P. JaAkkola, M. Ryynanen, W. H. Pearce, J. S. T. Yao, K. Majamaa, S. N. Smullens, Z. Gatalica, R. E. Ferrell, S. A. Jimenez, C. E. Jackson, V. V. Michels, M. Kaye \& H. Kuivaniemi. 1993. Sequencing of cDNA from 50 unrelated patients reveals that mutations in the triple-helical domain of type III procollagen are an infrequent cause of aortic aneurysms. J. Clin. Invest. 91: 2539-2545.

43. TILSON, M. D. 1988. Histochemistry of aortic elastin in patients with non-specific abdominal aortic aneurysmal disease. Arch. Surg. 123: 503-505.

44. Curran, M. E., D. L. Atkinson, A. K. Ewart, C. A. Morris, M. F. Leppert \& M. T. Keating. 1993. The elastin gene is disrupted by a translocation associated with supravalvular aortic stenosis. Cell 73: 159-168.

45. Olson, T. M., V. V. Michels, Z. Urban, K. Csiszar, A. M. Christiano, D. J. Driscoll, R. H. Feldt, C. D. Boyd \& S. N. Thibodeau. 1995. A 30 kb deletion within the elastin gene results in familial supravalvular aortic stenosis. Hum. Mol. Genet. 4: 1677-1679.

46. Ewart, A. K., C. A. Morris, D. Atkinson, W. Jin, K. Sternes, P. Spallone, A. D. Stock, M. Leppert \& M. T. Keating. 1993. Hemizygosity at the elastin locus in a developmental disorder, Williams syndrome. Nature Genet. 5: 11-16. 
47. Dietz, H. C., G. R. Cutting, R. E. Pyeritz, C. L. Maslen, L. Y. Sackai, G. M. Corson, E. G. Puffenterger, A. Hamosh, E. J. Nanthuakumar, S. M. Curristin, G. Stetten, D. A. Meyers \& C. A. Francomano. 1991. Marfan syndrome caused by a recurrent de novo missense mutation in the fibrillin gene. Nature 35e: 337-339.

48. Tsipouras, P., R. Del Mastro, M. Sarfarazi, B. Lee, E. Vitale, A. H. Child, M. Godfrey, R. B. Devereux, D. Hewett, B. Steinmann, D. Viljoen, B. C. Sykes, M. Kirkpatrick, F. Ramirez \& The International Marfan Syndrome CollaboRative Study. 1992. Genetic linkage of the Marfan syndrome, ectopia lentis, and congenital contractural arachnodactyly to the fibrillin genes on chromosome 15 and 5. N. Engl. J. Med. 326: 905-909.

49. St Jean, P. L., X. C. Zhang, B. K. Hart, H. Lamlum, M. W. Webster, D. L. Steed, A. M. Henney \& R. E. Ferrell. 1995. Characterization of a dinucleotide repeat in the $92 \mathrm{kDa}$ type IV collagenase gene (CLG4B), localization of CLG4B to chromosome 20 and the role of CLG4B in aortic aneurysmal disease. Ann. Hum. Genet. 59: $17-24$.

50. Huhtal.a, P., A. Tuuttila, L. T. Chow, J. Lohi, J. Keski-Oja \& K. Tryggvason. 1991. Complete structure of the human gene for 92-kDa type IV collagenase: Divergent regulation of expression for the 92- and 72-kilodalton enzyme genes in HT-1080 cells. J. Biol. Chem. 266: 16485-16490.

51. Formstone, C. J., P. J. Byrd, H. J. Ambrose, J. H. Riley, D. Hernandez, C. M. MCCONVILLE \& A. M. R. TAYLOR. 1993. The order and orientation of a cluster of metalloproteinase genes, stromelysin 2 , collagenase, and stromelysin, together with D11S385, on chromosome 11q22-q23. Genomics 16: 289-291.

52. Freije, J. M. P., I. Diez-Itza, M. Balbin, L. M. Sanchez, R. Blasco, J. Tolivia \& C. Lopez-OTIN. 1994. Molecular cloning and expression of collagenase-3, a novel human matrix metalloproteinase produced by breast carcinomas. J. Biol. Chem. 269: 16766-16773.

53. Pendas, A. M., T. Matilla, X. Estivill \& C. Lopez-Otin. 1995. The human collagenase-3 (CLG3) gene is located on chromosome 11 q22.3 clustered to other members of the matrix metalloproteinase gene family. Genomics 26: 615-618. 